\title{
Lire et visiter la France de Balzac. Touristes et écrivains anglophones en France provinciale, 1880-1920
}

Reading and Visiting Balzac's France. Anglophone Tourists and Writers in Provincial France, 1880-1920

\section{Tom Williams}

\section{OpenEdition Journals}

Édition électronique

URL : https://journals.openedition.org/recherchestravaux/2211

DOI : 10.4000/recherchestravaux.2211

ISSN : 1969-6434

Éditeur

UGA Éditions/Université Grenoble Alpes

\section{Édition imprimée}

ISBN : 978-2-37747-197-3

ISSN : 0151-1874

Référence électronique

Tom Williams, « Lire et visiter la France de Balzac. Touristes et écrivains anglophones en France provinciale, 1880-1920», Recherches \& Travaux [En ligne], 96 | 2020, mis en ligne le 23 juin 2020, consulté le 30 juin 2021. URL : http://journals.openedition.org/recherchestravaux/2211 ; DOI : https:// doi.org/10.4000/recherchestravaux.2211

Ce document a été généré automatiquement le 30 juin 2021.

(c) Recherches \& Travaux 


\title{
Lire et visiter la France de Balzac. Touristes et écrivains anglophones en France provinciale, 1880-1920
}

\author{
Reading and Visiting Balzac's France. Anglophone Tourists and Writers in \\ Provincial France, 1880-1920
}

Tom Williams

1 En octobre 1913, un article publié dans The Times chante les louanges de la France provinciale et déplore le fait, qu'à l'exception de Paris, les villes françaises demeurent aussi peu connues par les touristes britanniques :
On ne peut pas comprendre la France si on n'a pas encore [...] étudié les Français dans les villes de province, si on n'a pas encore, pour reprendre des exemples de Balzac, ce maitre de l'esprit et des mœurs provinciales, noué des amitiés parmi Les Célibataires, ou si on n'a jamais été, même temporairement, chez soi dans une retraite comme La Grenadière ${ }^{1}$.

2 Pour ce journaliste, la « vraie » France se trouverait ainsi en province, et les œuvres de Balzac constitueraient un guide incontournable pour connaître, ou pour reconnaître, cette France authentique.

Le présent article analyse l'intérêt porté par des voyageurs et écrivains britanniques et américains aux œuvres de Balzac et aux lieux qui lui sont associés. Il interroge la relation tissée, dans leurs récits de voyage, entre les textes littéraires et lieux visités, et la place des œuvres de Balzac dans la construction des expériences de voyage dans la France provinciale. Entre les années 1880 et 1920, l'essor du tourisme en général, et du tourisme littéraire en particulier, coïncide avec l'apogée de la réputation de Balzac dans le monde anglophone. En effet, si Balzac a ses premiers admirateurs outre-manche dès les années 1830 , ce n'est qu'à partir de la fin des années 1870 que ses romans, considérés comme scandaleux par le public victorien, deviennent non seulement acceptables mais aussi salués par la critique ${ }^{2}$. Les références à Balzac dans les écrits de voyage sont alors fréquentes et témoignent à la fois de la popularité de Balzac en tant 
qu'auteur et du fait que son œuvre constitue l'une des seules sources de connaissance de la France provinciale pour le lecteur anglophone.

4 Les expériences de ces écrivains anglophones ont ainsi été façonnées non seulement par ce qu'ils ont vu et entendu pendant leurs voyages, mais aussi par ce qu'ils avaient lu avant et pendant leurs visites. Seront dès lors étudiées ici l'importance des sites associés à la vie et aux œuvres de Balzac comme lieux de "pèlerinage littéraire » ainsi que les pratiques de lecture des voyageurs, et la manière dont leurs attentes et leurs expériences ont été enrichies par la connaissance des œuvres. Après une présentation générale de la place de Balzac dans le tourisme littéraire anglophone de 1880 à 1920, cet article analysera l'influence de ses œuvres sur les expériences de voyage de deux auteurs : Henry James, fortement influencé par Balzac en tant que romancier, et qui commence son Petit tour en France de 1883 par la visite de la maison natale de Balzac à Tours, et Matilda Betham Edwards, romancière et francophile britannique, qui publie notamment des descriptions de ses voyages à la recherche de Balzac dans son livre de 1909, Promenades littéraires en France ${ }^{3}$. Ni James, ni Betham Edwards n'ont circonscrit leurs « pèlerinages littéraires » à Balzac: James visite des sites associés à de nombreux autres écrivains, dont Pétrarque, Rabelais, Stendhal et Rousseau, tandis que Betham Edwards consacre des chapitres de ses Promenades littéraires à Flaubert et à Mérimée, et décrit en détail un "pèlerinage » à la maison de George Sand à Nohant. Néanmoins, Balzac et ses œuvres constituent un point de référence majeur pour ces deux auteurs, une clé d'entrée pour leur exploration de la France provinciale.

\section{Balzac dans le tourisme littéraire anglophone : textes, lieux et paysages}

5 Le tourisme littéraire connaît un essor marqué au cours du XIX ${ }^{e}$ siècle. Pendant cette période, selon Nicola Watson, «l'habitude de visiter des lieux associés à des livres particuliers afin de savourer le texte, le lieu et leurs relations mutuelles, est devenue un phénomène commercialement important ${ }^{4} »$. Au Royaume-Uni, le Stratford de Shakespeare, l'Abbotsford de Scott et le Haworth des sœurs Brontë deviennent ainsi des lieux fameux de "pèlerinage littéraire". La maison natale des écrivains, leurs résidences, leurs tombes et les monuments érigés en leur honneur attirent des touristes, tout comme les paysages ou les régions plus vastes où se déroulent leurs œuvres. Sous le titre de Géographie littéraire, William Sharp décrit en 1904 une série de paysages rendus célèbres par la littérature, comme par exemple «le pays de George Eliot », « Dickens-Land » et "Scott-Land ${ }^{5}$ ». Selon Watson, cette exploration de paysages ou de régions littéraires atteint son apogée entre les années 1880 et 1920, années où "la France de Balzac» suscite un vif intérêt parmi des voyageurs anglophones ${ }^{6}$.

6 Si le tourisme littéraire est le plus souvent perçu, à l'instar de Watson, comme un phénomène par lequel les écrivains sont célébrés et commémorés dans un cadre national, il franchit parfois les frontières nationales. L'Américain Milburg Francisco Mansfield, qui écrit sous le pseudonyme de Frances Miltoun, publie ainsi un des premiers guides sur le Londres de Dickens en 1903, suivi un an plus tard par un guide sur le Paris d'Alexandre Dumas ${ }^{7}$. Arthur Bartlett Maurice, qui a publié une chronique consacrée au tourisme littéraire dans la revue américaine The Bookman sous le titre « Le Baedeker littéraire », rédige un guide du New York des romanciers en 1915, suivi en 1919 
par un guide de Paris dans lequel il affirme qu' " aucune ville et aucun pays n'étaient aussi riches en lieux de pèlerinage littéraire que Paris et la France ", évoquant, au-delà de la capitale, les sites associés à Flaubert, Maupassant, Sue, Sand ou encore Zola ${ }^{8}$. Dans son guide de Paris, Maurice consacre chaque chapitre à un auteur différent, décrivant ainsi « le Paris de Victor Hugo » ou encore «le Paris d'Alphonse Daudet », une pratique déjà établie dans un guide publié par Benjamin Ellis Martin et Charlotte Martin en 1899, Les Pierres de Paris, qui propose lui aussi aux lecteurs de découvrir «le Paris d'Alexandre Dumas", «le Paris d'Honoré de Balzac» ou encore «le Paris de Victor Hugo ${ }^{9}$ ». Tandis que des recensions de ces deux livres soulignent que «le Paris de Balzac » aurait mérité un livre entier, aucun guide de ce genre n'est alors publié ${ }^{10}$. Si Paris demeure de loin le lieu de prédilection du tourisme international, d'autres sites et paysages littéraires, en province, sont visités par les touristes anglophones. Dans son livre sur la Normandie, Miltoun estime que « tous ceux qui viennent à Rouen devraient accomplir un pèlerinage à la maison de Flaubert $^{11}$ ", tandis que la romancière américaine Edith Wharton effectue son propre pèlerinage au château de Nohant, décrivant la campagne environnante comme " le pays de George Sand », et se réjouit de découvrir aux portes du château une jeune fille qui aurait pu "sortir tout droit » d'un des romans de l'écrivaine ${ }^{12}$.

7 Balzac constitue alors une référence littéraire primordiale pour les voyageurs anglophones en quête de la France provinciale. Avant de les visiter, de nombreux lecteurs anglophones ne connaissent certaines villes de province que par la lecture de Balzac. Le critique Arthur Bingham Walkley écrit ainsi en 1889 que la ville de Tours "nous serait complètement inconnue si elle n'avait été le lieu de résidence de l'Abbé Birotteau et de Mlle Gamard. De même, le fervent balzacien ne connaît Douai que comme la ville de Balthazar Claes ; Limoges, celle de Mme Graslin ; Angoulême, celle de Lucien de Rubempré ; Sancerre, celle de Mme De la Baudraye ${ }^{13}$ ». En vertu de la minutie des descriptions, les œuvres de Balzac sont en effet considérées presque comme des guides touristiques en elles-mêmes et sont recommandées aux voyageurs ${ }^{14}$. L'Américain Francis Miltoun, dans un livre sur les châteaux de la Loire, note par exemple que «Le Lys dans la Vallée de Balzac donnera une vision plus charmante de la Touraine ancienne que toute une série de guides touristiques et de livres d'histoire arides ${ }^{15}$ ».

8 À mesure que le chemin de fer puis l'automobile rendent les provinces françaises plus accessibles aux touristes étrangers, l'intérêt pour ces formes de pèlerinage littéraire croît. En 1906, Francis Miltoun qualifie la maison dans laquelle Balzac naquit à Tours de "principal lieu de pèlerinage littéraire à Tours" et observe que des "pèlerins littéraires » visitent Saumur en raison de ses liens avec Eugénie Grandet ${ }^{16}$. Plusieurs voyageurs expriment explicitement leur excitation à l'idée de visiter des lieux qui leur sont déjà familiers grâce à la littérature et sont convaincus que ces références sont partagées par tout lecteur éduqué. Dans son ouvrage paru en 1909, À travers les provinces françaises, l'artiste et écrivain américain Ernest Peixotto rapporte que lors de la visite de Nemours, il ne put "s'empêcher de penser à la petite Ursule Mirouet ${ }^{17}$ ». En poursuivant son périple, à Limoges, il prend plaisir à observer la vue depuis le nouveau pont sur la Vienne, « la vue sur laquelle Balzac s'attarde avec un plaisir si évident dans son Curé de village ${ }^{18}$ ». De même, en admirant la vue depuis le train reliant Tours et Chinon, Theodore Andrea Cook se réfère à Balzac à plusieurs reprises en contemplant "le pays où Félix trouva son "Lys dans la Vallée ${ }^{19 "}$ ". " Dans nos voyages en Bretagne on peut identifier les Du Guénic, le vieux Baron, Mlle Zéphirine, Calyste et Gasselin, et tous les autres personnages que Balzac, dans son roman, a si admirablement dépeints ${ }^{20}$ ", 
considère à son tour l'Américain Ange Mosher dans Le charme de la Bretagne. La connaissance des œuvres de Balzac influe ainsi sur la perception par les touristes anglophones des provinces françaises, mais aussi de leurs habitants, même si les œuvres de Balzac ont été écrites plus qu'un demi-siècle auparavant.

9 Si ces nombreuses références à Balzac illustrent la manière dont l'expérience du voyage a pu être enrichie par le souvenir d'œuvres littéraires, le récit de voyage publié en 1909 par le Britannique Frederic Lees, Un été en Touraine, invite le voyageur à lire les œuvres de Balzac in situ afin de mieux apprécier la visite des lieux : " Il est du devoir de tous ceux qui voyagent en Touraine de renouer connaissance avec certaines des histoires du grand romancier ", explique Lees après son arrivée à Tours. "Elles présentent un intérêt supplémentaire quand elles sont lues au milieu des beautés naturelles parmi lesquelles elles ont été conçues ». Lire les œuvres de Balzac in situ constitue aux yeux de Lees "une excellente méthode pour se mettre au diapason de son environnement", même s'il admet que les descriptions n'ont « que peu de valeur pratique » et qu'il n'a «tiré que de maigres informations topographiques ou historiques de ces livres ${ }^{21}$ ».

Pour le lecteur à la recherche d'informations topographiques supplémentaires, ou pour celui qui n'a ni le temps ni l'espace dans ses bagages pour se consacrer à la lecture de romans entiers, l'éditeur de guides de voyage August Hare propose un guide pratique et maniable juxtaposant des informations sur les villes, les transports ou les hôtels avec des morceaux choisis de romans, de récits de voyage ou d'œuvres historiques. Dans le guide Le Sud-Ouest de la France publié en 1890, Hare accompagne la description des villes de Saumur et Guérande d'extraits d'environ deux pages d'Eugénie Grandet et de Béatrix ${ }^{22}$. La présentation des textes littéraires et de leur importance est très succincte, et se résume à la une seule note de bas de page recommandant aux «visiteurs de Guérande [de] lire Béatrix de Balzac ", même s'il "n'est pas facile d'y trouver une maison qui corresponde exactement à celle décrite par Balzac comme la résidence du baron de Guénic $^{23}$ ». Dans son guide pour Le Nord-Ouest de la France, Hare introduit la ville de Fougères par un long extrait des Chouans de Balzac, mais prend garde à limiter les attentes du visiteur : " La description de Balzac donnerait sans doute des attentes trop élevées, mais cela vaut la peine de la lire sur place ${ }^{24}$. »

11 Le vif intérêt des voyageurs anglophones pour les sites associés à Balzac s'immisce également dans la fiction. Une quinzaine de printemps en France, un curieux livre combinant roman et guide touristique, met en scène un personnage principal qui « détestait quitter Saumur sans avoir trouvé la maison que Balzac avait choisie pour Eugénie Grandet, dans ce roman si puissant mais si sordide ${ }^{25}$ ». Avec humour, le récit de voyage fictif Le paratonnerre, mettant en scène un touriste américain traversant la France en automobile, pousse le tourisme littéraire balzacien aux limites du ridicule :

J'ai lu Balzac. Je ne l'ai jamais apprécié comme je le fais ici, dans son «pays natal». J'ai supplié Brown de nommer la voiture de son maître «Balzac », car elle est elleaussi un "génie violent et compliqué ». J'ai regardé la maison où est né Balzac, j’ai photographié le médaillon Balzac, j'ai bourré mon coffre d'éditions illustrées des livres de Balzac, et je me suis attaché à aller trouver tout ce dont il a jamais parlée ${ }^{26}$.

12 Cette histoire "fort charmante mais sans importance" est selon Arthur Bartlett Maurice dans The Bookman, si populaire que « des vingtaines de lecteurs américains ont été incités à suivre en automobile l'aventure des personnages dans l'histoire ». Tout cela prouve, selon lui, qu'il n'est « absolument pas nécessaire que le pèlerin prenne sa fiction au sérieux pour que le pèlerinage littéraire soit un plaisir ${ }^{27}$ ». Si cela est sans doute vrai pour les admirateurs du Paratonnerre, la plupart des pèlerins à la recherche 
de la France de Balzac prennent les œuvres de ce dernier très au sérieux. C'est particulièrement évident pour Henry James.

\section{Henry James : le touriste sentimental}

13 Peu d'écrivains du monde anglophone étaient aussi admiratifs de Balzac et si fortement inspirés par ses œuvres qu'Henry James (1843-1916), qui consacre cinq essais à l'auteur de La Comédie humaine, soit plus qu'il n'écrit sur n'importe quel autre écrivain ${ }^{28}$. Son essai de 1875 joue un rôle important dans le rétablissement de la réputation de Balzac au cours des dernières décennies du $\mathrm{xIX}^{\mathrm{e}}$ siècle $^{29}$. Balzac est pour James le modèle du romancier, et plusieurs de ses propres romans révèlent des parallèles avec ses œuvres ${ }^{30}$. James admire en effet chez Balzac la description minutieuse des lieux géographiques, des bâtiments et des objets, et déclare qu'il «n'y a rien d'autre dans la littérature imaginative qui ressemble à sa puissante passion pour les choses ${ }^{31}$ ». Il exprime même parfois sa frustration de ne pas pouvoir saisir le caractère d'un cadre géographique et le reproduire avec la même habileté que Balzac. En 1907, par exemple, James affirme avoir pour Roderick Hudson, son roman publié en 1875, travaillé «dans l'ombre imposante » de Balzac et s'être efforcé de dépeindre une ville de province américaine de la même manière que Balzac le faisait pour Saumur, Limoges ou Guérande ${ }^{32}$.

Il est difficile de séparer, dans la vie et l'œuvre de Henry James, sa fascination pour la France de celle pour Balzac ${ }^{33}$. Ce serait en effet l'attraction pour le Paris de Balzac qui aurait motivé son séjour dans la capitale française en 1875-1876 ${ }^{34}$. En octobre 1882, il part à la recherche de la France provinciale, un voyage qu'il raconte ensuite dans une série d'articles parus dans The Atlantic Monthly sous le titre français "En province " avant de les publier en 1884 dans un livre intitulé Un petit tour en France. Ce récit exprime son désir de voir la France au-delà de Paris, contrairement à ses contemporains américains qui ont selon lui «trop tendance à identifier la France à Paris $^{35}$ ». Mais il ne prétend cependant pas découvrir lui-même la province car «si quelqu'un a découvert la province ou, du moins, l'a révélée, ce fut Balzac ${ }^{36} »$. Son voyage commence en Touraine, qu'il considère comme la province la plus " française ", notamment en raison des écrivains qui lui sont associés: "La Normandie est la Normandie, la Bourgogne est la Bourgogne, la Provence est la Provence, mais la Touraine est essentiellement la France. C'est le pays de Rabelais, de Descartes, de Balzac $^{37}$ ", écrit-il. Fortement imprégné par la littérature française, James voyage donc à la recherche de ce qu'il considère comme "essentiellement la France ", espérant rencontrer des scènes et des paysages pittoresques qui symbolisent pour lui l'image authentique de la France provinciale.

15 En tant qu'auteur de récits de voyage, Henry James se présente comme un «touriste sentimental », racontant non seulement ce qu'il voit, mais aussi les sentiments inspirés par sa rencontre avec la France. Mais il est également touriste littéraire, visitant des sites associés à des auteurs et à leurs œuvres, expliquant fréquemment comment les représentations littéraires d'un lieu lui donnent des significations supplémentaires et façonnent sa propre expérience de touriste ${ }^{38}$. La pratique du tourisme littéraire figure dans la grande majorité des récits de voyage de James. Ses voyages en Angleterre, par exemple, le conduisent à la statue de Samuel Johnson à Lichfield, au mémorial de Robert Browning à l'abbaye de Westminster et au « pays de Shakespeare », où il observe les foules de touristes américains se rendant à Stratford-on-Avon ${ }^{39}$. Sa visite d'un 
hospice paroissial (workhouse) anglais en 1879 le transporte « dans les premières pages d'Oliver Twist ${ }^{40}$ ». De même, ses voyages en Italie le conduisent à la tombe de Dante et à la maison de Byron à Ravenne, tandis que son expérience de la ville d'Assise est altérée par le souvenir d'une description de Goethe ${ }^{41}$. Lors de son séjour en France, le souvenir d'un poème de Matthew Arnold sur la ville de Cette (aujourd'hui Sète) suffit à changer son impression d'un lieu qu'il considérerait autrement comme sans grand intérêt : «[...] cette strophe de Matthew Arnold, dont je me souvenais par hasard, a donné une certaine importance à la demi-heure que je passai au buffet de la gare de Cette en attendant le train pour Montpellier ${ }^{42}$ ». James effectue également un "pèlerinage » à l'église de Brou à Bourg-en-Bresse, qu'il ne connaît que par un poème d'Arnold. Il se rappelle alors l'« endroit où [il] l'avait lu pour la première fois, et où [il] l'avait relu et relu en demandant si [il] aurait un jour la chance de visiter l'église de Brou $^{43}$ ». Si ses souvenirs du poème ne correspondent pas tout à fait son expérience de voyage, cela ne l'empêche pas « de ressentir qu'[il] regardait un monument dont Matthew Arnold était en partie responsable, du moins quant à l'effet qu'il produisait sur l'esprit du touriste d'aujourd'hui ${ }^{44}$ ». Henry James est également convaincu que Balzac est «en partie responsable » de la manière dont il perçoit les lieux associés à sa vie et à ses œuvres.

James commence son voyage à Tours, où il s'interroge longuement sur l'incongruité de la maison natale de Balzac, si modeste et relativement récente, et où l'absence d'un monument à l'auteur de La Comédie humaine le surprend: ce n'est en effet que trois ans après la visite de James que le conseil municipal vote la construction d'un monument, finalement inauguré en 1889. Il ne peut dissocier la Touraine de Balzac: il existe selon lui « certaines correspondances entre son caractère et celui de sa province natale » et Balzac, le produit de ce terroir historique, est pour lui "pénétré du sentiment du passé $^{45} »$. C'est surtout en raison des références littéraires qu'il lui associe que Tours présente pour James un intérêt. L'admiration pour l'architecture de la cathédrale de Tours cède rapidement la place à un souvenir littéraire et James part à la recherche de la maison de Mademoiselle Gamard dans Le Curé de Tours, dans laquelle logea l'Abbé Birotteau. Tandis que James admet qu'il devrait peut-être avoir honte d'avouer que «le nom profane de Balzac» a donné pour lui un intérêt supplémentaire à ce "vénérable sanctuaire ${ }^{46}$ ", ses descriptions de la cathédrale reproduisent finalement celle de Balzac, que James trouve plus intéressante que la réalité elle-même. Comme le note David Spurr, "la cathédrale semble plus réelle et acquiert sa véritable signification pour James dans la nouvelle de Balzac, et non dans sa présence physique ${ }^{47}$ ». À plusieurs reprises, la connexion balzacienne justifie à elle seule la visite d'un lieu. C'est ainsi qu'il décide de se rendre à Langeais avant tout autre château de la Loire, car il se souvient « de la duchesse de Langeais, qui figure dans plusieurs romans de Balzac et qui lui confère un pouvoir évocateur très puissant ${ }^{48}$ ».

Pour autant, si James rapporte que le simple fait que Balzac ait décrit un lieu situé à proximité suffit à éveiller son intérêt, il ne visite pas l'intégralité de ceux-ci. S'il note par exemple que le manoir de Clochegourde, résidence de Mme de Mortsauf, l'héroïne du Lys dans la vallée, "a probablement pour modèle un original que l'on pourrait retrouver aujourd'hui », il ne s'y rend pas en dépit de la proximité de Tours: «Il y a tant de châteaux en Touraine que l'histoire a rendus célèbres, et l'on n'en finirait plus s'il fallait rechercher tous ceux auxquels la fiction a donné un nom ${ }^{49}$ ». De même, quand son train traverse la ville d'Angoulême, qu'il identifie à tort comme « la vieille capitale de l'Anjou », James observe qu'il aurait dû s'y arrêter "en mémoire de David et 
Ève Séchard, de Lucien de Rubempré et de Mme de Bargeton », mais il ne descend pas du train, par peur d'être déçu par la réalité :

Les personnages que je viens de citer n'ont pas l'identité floue qui afflige les personnages historiques; ils sont réels, suprêmement réels, car ils sont les fils du grand Balzac qui leur a inventé une réalité artificielle infiniment supérieure à la réalité vulgaire ${ }^{50}$.

Autrement dit, l'expérience concrète de la visite d'un lieu ne pouvait rivaliser pour James avec la réalité fictive fabriquée par Balzac.

Tout en avouant qu'il regrette de ne pas avoir vu à Angoulême «les jolies vues dont Balzac donne la description ", il justifie sa décision par une autre référence à Balzac : "La vérité est que je n'avais aucun besoin, à cet instant précis, d'entrer en communication avec Balzac car j'avais en face de moi, dans le compartiment, deux personnages presque aussi remarquables que les acteurs de La Comédie humaine ${ }^{51} »$. Une telle remarque est typique de James, qui juge les personnes rencontrées au cours de ses voyages en fonction de leur degré de correspondance avec les attentes créées par sa lecture d'œuvres littéraires. De même, lors d'un voyage à Québec en 1871, James décrit le caractère "provincial français " de la ville en se référant à Balzac, notant par exemple que "les soirées ici devaient être aussi ennuyeuses que celles décrites par Balzac dans sa Vie de Province » ou encore qu'il avait « rencontré dans les rues d'ici, des petits vieux Français qui ont l'air d'être sortis de Balzac - rongés des habitudes de classe, ridés par les expressions du vieux monde ${ }^{52}$ ». Le sens du pittoresque chez James, comme sa recherche d'une image authentique de la France provinciale, reposent sur les attentes élevées acquises comme lecteur de la littérature française.

Ces attentes sont également susceptibles d'entraîner des désillusions. À Bourges, James exprime sa déception de ne pas être confronté à des personnalités balzaciennes : " Je n'en ai vu aucun qui m'ait fait penser à "l'illustre Gaudissart" de Balzac; et en réalité, au cours de mon mois de voyage à travers une grande partie de la France, j'ai entendu si peu de conversations décousues que je me suis demandé s'il n'y avait pas eu un changement dans l'esprit de ce peuple ${ }^{53}$ ". Plus généralement, ce voyage à travers la France provinciale se révèle une déconvenue car s'il confie à Grace Norton trouver la Touraine « charmante ", il juge les autres régions françaises " plutôt décevantes ${ }^{54}$ ». Il regrette avant tout le défaut de pittoresque, comme il l'écrit à son frère, William :

Je poursuis mon pèlerinage à travers ces villes françaises plutôt ennuyeuses. [...]

C'est un travail plutôt monotone, car la plupart des endroits, je suis désolé de le

dire, sont beaucoup moins pittoresques que je ne l'avais imaginé 55 .

21 L'impossibilité de faire l'expérience concrète d'une France pittoresque, c'est-à-dire de cette France de Balzac fantasmée, conduit James à la désillusion: la réalité manque selon lui d'authenticité. Ainsi, chez James, l'expérience du voyageur entre parfois en contradiction avec ses attentes nées de la lecture des œuvres de Balzac. Si l'auteure anglaise Matilda Betham Edwards constate également certains contrastes entre les œuvres littéraires et la réalité des lieux, ni son enthousiasme pour le tourisme ni sa passion pour la littérature française n'en sont atténués.

\section{Matilda Betham Edwards : la pèlerine littéraire}

Romancière et auteure prolifique de récits de voyage, Matilda Betham Edwards (1836-1919) revendique l'enrichissement apporté par la lecture des œuvres Balzac à 
l'expérience du voyage. Des années 1870 à la Première Guerre mondiale, Betham Edwards publie de nombreux ouvrages sur la France de la Troisième République, jouant ainsi un rôle important d'interprète auprès des lecteurs anglophones. Son travail est récompensé par l'octroi du titre d'Officier de l'Instruction Publique en $1891^{56}$. Comme Henry James, Betham Edwards soutient dans ses œuvres l'idée que la France ne se réduit pas à Paris, et qu'il importe de délaisser les endroits habituellement les plus fréquentés pour découvrir des régions « totalement inconnues de la plupart des touristes ${ }^{57}$ ».

Alors que, contrairement au voyageur sentimental Henry James, les récits de voyage de Matilda Betham Edwards sont marqués surtout par une volonté d'informer et d'éduquer le public anglophone, elle se considère néanmoins comme une "pèlerine littéraire ». Son premier livre de voyage décrit ainsi un séjour de deux mois à Weimar où elle visite, comme tant d'autres "pèlerins ", des lieux associés à la vie de Goethe et de Schiller ${ }^{58}$. Pourtant, son premier livre sur la France, publié en 1877 sous le titre Une année dans l'Ouest de la France, qui relate un long séjour effectué à Nantes en 1875 à la maison du médecin et homme politique Ange Guépin, contient peu de réflexions sur la littérature française, et se soucie avant tout de donner une vision positive de l'évolution politique de la France depuis la fondation de la Troisième République ${ }^{59}$. Une recension de ce premier livre, publiée dans le Pall Mall Gazette en 1877, critique d'ailleurs son manque de culture littéraire : «Visiter Guérande, Le Croisic et Escoublac et ne pas penser à l'histoire la plus passionnée de Balzac, Béatrix, ni même se souvenir

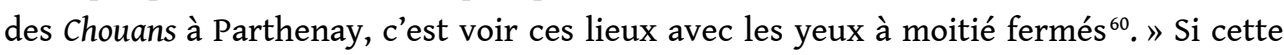
remarque ne représente que l'opinion d'un seul critique, on peut imaginer que d'autres lecteurs anglophones, qui n'avaient découvert ces lieux qu'à travers la lecture des œuvres de Balzac, furent déçus de ne pas retrouver ces connexions dans le premier récit de voyage de Betham Edwards.

Cette lacune fut comblée dans ses œuvres ultérieures sur la France, où les références à Balzac abondent. Publiées à la fin du siècle, ses Réminiscences racontent, par exemple, son premier séjour à Paris dans une pension où, selon elle, «tout ressemblait autant à un chapitre du Cousin Pons ou du Père Goriot qu'il était possible de l'imaginer ${ }^{61} »$. En se remémorant d'autres épisodes de sa vie, elle fait régulièrement appel à Balzac: elle raconte avoir assisté à "des scènes qui [lui] rappelaient Balzac", avoir entendu des histoires à partir desquelles "Balzac aurait fait un chef-d'œuvre», et avoir vu des " scènes et des épisodes auxquels seul Balzac pourrait faire justice ${ }^{62}$ ». La Vie en France, son étude de 1905 sur les classes sociales et la vie familiale françaises est remplie de références à Balzac "ce délinéateur encyclopédique de la vie française ", tandis qu'elle publie la même année une étude de cinquante pages sur la relation entre Balzac et Ewelina Hanska ${ }^{63}$.

Dans ses Promenades littéraires en France, un recueil d'articles précédemment parus dans la presse périodique (et notamment l'article de «Sur la piste de Balzac » publié dans The Sphere en 1904) $)^{64}$ puis sous forme de livre en 1907, Betham Edwards mentionne régulièrement la présence dans certaines villes du Val de Loire d'un tourisme littéraire associé aux œuvres de Balzac : « Saumur, la maison d'Eugénie Grandet, et Guérande, la scène de Béatrix, sont, en effet, devenus des lieux de pèlerinage littéraires ${ }^{65} \%$. Dans le cas de Saumur, Betham Edwards suggère que les références littéraires de la ville sont devenues encore plus importantes pour les touristes que son passé historique : 
Qui, en visitant la belle petite ville de Saumur, pense aux personnages historiques qui y sont liés ? Même le grand personnage de Duplessis Morny est éclipsé par celui de la fille de l'avare, la douce et malheureuse Eugénie Grandet ${ }^{66}$.

Contrairement à Lees, en revanche, elle revendique l'utilité pratique de ces livres qui constitueraient en eux-mêmes des guides touristiques: «Il y a certaines villes françaises pour lesquelles Balzac est le meilleur guide possible, voire un guide indispensable ${ }^{71}$ ». En décrivant l'évêché de Limoges, par exemple, elle cite un long extrait du Curé de village qui aurait pu, selon elle, "être écrit à l'usage des voyageurs » et dans lequel « tous les sites semblent aussi identifiables qu'ils le sont dans des guides touristiques $^{72}$ ». Elle va même jusqu'à suggérer que certains passages très descriptifs de Balzac seraient encore plus intéressants pour le touriste, pour lequel ils ont un « intérêt vital » que pour le lecteur lambda, qui pourrait trouver la minutie des détails lassante ${ }^{73}$. , pour Betham Edwards, la France de Balzac est encore identifiable à plus de cinquante ans de distance. En dépit de certains effets visibles de la modernisation, elle rassure ses lecteurs sur la modestie des évolutions depuis l'époque de Balzac. À Limoges, par exemple, elle prend plaisir à observer « en se promenant dans la ville basse avec le roman de Balzac à la main [...] que ces rues tortueuses et demeures anciennes doivent rester comme il les a vues il y a trois quarts de siècle ${ }^{74}$ ». À Nemours, son constat est presque identique, tout en attirant l'attention du lecteur sur les changements technologiques qui ont, entre autres, ouvert les provinces au tourisme à la fin du XIX ${ }^{e}$ siècle : «En nous promenant dans les rues herbeuses, nous pensons que les chemins de fer, les téléphones et le reste n'ont que très peu changé Nemours depuis les 
descriptions de Balzac, écrites il y a trois quarts de siècle ${ }^{75}$. « « La Guérande décrite par Balzac était toujours présente » observe-t-elle en se remémorant un voyage antérieur à Guérande, malgré le fait que « les Haussmann locaux étaient déjà au travail et que la modernité l'avait décloisonné[e] de son ancienne solitude ». La mémoire imprégnée par le roman Béatrix, elle affirme qu'elle fut, dès son entrée en ville, "immédiatement ramenée à l'année $1836^{76}$ ». Visiter la France de Balzac constitue ainsi pour Betham Edwards un voyage dans le temps, comme si l'âge de Balzac représentait la période historique la plus authentique et intéressante pour le voyageur. Ainsi, l'essor du tourisme littéraire balzacien à partir des années 1880 résulte en partie de la persistance paradoxale d'une France semblant issue d'une époque antérieure rendue plus visible et accessible aux touristes par les transports modernes.

La période entre les années 1880 et 1920 semble représenter un âge d'or du tourisme littéraire en général et du tourisme balzacien anglophone en particulier. Pendant cette période, la réputation littéraire de Balzac connaît son apogée, le chemin de fer et l'automobile rendent la France provinciale plus accessible et, celle-ci, en dépit de profondes transformations, présente toujours une ressemblance marquée avec le monde décrit par Balzac un demi-siècle auparavant. Dans ce contexte, Henry James et Matilda Betham Edwards, tout comme Frederic Lees, Francis Miltoun et de nombreux autres écrivains anglophones partent à la recherche de la "France de Balzac» et proposent une réflexion approfondie sur les relations entre la lecture d'œuvres littéraires, la visite des lieux et l'expérience du voyageur. Le fait qu'ils aient connu ces lieux en tant que lecteurs avant de les visiter en tant que touristes crée inévitablement un décalage entre leurs attentes et la réalité. Que cela conduise à une certaine déception, comme dans le cas de Henry James ou à une sorte d'enrichissement mutuellement bénéfique comme dans le cas de Betham Edwards, leurs expériences de lecture transforment régulièrement leurs expériences des lieux réels en tant que voyageurs, au point que les imaginaires littéraires associés à un lieu pourraient susciter un intérêt encore plus grand que la réalité.

31 Seule une étude entière permettrait de saisir pourquoi l'intérêt pour le tourisme littéraire balzacien semble décroître dans la littérature de voyage anglophone à partir des années 1920. Plusieurs facteurs peuvent cependant être suggérés, au-delà du déclin d'intérêt porté à cet auteur qui incarne de plus en plus un monde lointain et, du point de vue du voyageur, de plus en plus difficile à retrouver. Le déclin plus général d'une culture commémorative centrée sur les grands hommes de génie littéraire ou encore l'avènement du tourisme de masse sonnent tout d'abord le glas d'une époque où la bonne connaissance par les lecteurs des chefs-d'œuvre de la littérature française relevait de l'évidence pour l'auteur d'un guide ou d'un récit de voyage. De même, le déclin du réalisme littéraire et l'avènement du modernisme mettent fin à une vision de la littérature comme reproduction fidèle de la réalité. Reflet de ce crépuscule du tourisme littéraire balzacien, le récit de voyage sur la Loire, publié en 1926 dans une collection britannique réputée de guides et de récits de voyage, consacre de longs chapitres aux villes de Tours et de Saumur, mais reste muet sur les liens entre Balzac et chacune de ces cités ${ }^{77}$. 


\section{NOTES}

1. "The Charm of Provincialism: A Holiday in France ", The Times, 8 octobre 1913, p. 9. Sauf mention contraire, toutes les citations sont traduites de l'anglais par l'auteur.

2. S. Monod, "La fortune de Balzac en Angleterre", Revue de littérature comparée, $\mathrm{n}^{\circ}$ 24,1950, p. 181-210 ; C.R. Decker, The Victorian Conscience, New York, Twayne Publishers, 1952, p. 49-61.

3. H. James, A Little Tour in France, Boston MA, James R. Osgood and Company, 1885 (les citations dans le présent article proviennent de l'édition française: H. James, Voyage en France, trad. de l'anglais par P. Blanchard, Paris, Robert Laffont, 1987) ; M. Betham Edwards, Literary Rambles in France, Londres, Archibald Constable and Company, 1907.

4. N. J. Watson, The Literary Tourist: Readers and Places in Romantic and Victorian Britain, Basingstoke, Palgrave Macmillan, 2006, p. 1.

5. W. Sharp, Literary Geography, Londres, Pall Mall Publications, 1904.

6. N. J. Watson, ouvr. cité, p. 169.

7. F. Miltoun, Dickens' London, Boston, L.C. Page, 1903. F. Miltoun, Dumas' Paris, Boston, L.C. Page, 1904.

8. A. B. Maurice, The New York of the Novelists, New York, Dodd, Mead and Co., 1915 ; A. B. Maurice, The Paris of the Novelists, New York, Doubleday, 1919, p. Xx.

9. B. E. Martin et C. M. Martin, The Stones of Paris, vol. 2, New York, Charles Scribner's Sons, 1899.

10. The Times, 4 mars 1920, p. 17 ; The Bookman, décembre 1900, p. 327.

11. F. Miltoun, Rambles in Normandy, Boston, L.C Page, 1906, p. 197.

12. E. Wharton, A Motor Flight through France, New York, Charles Scribner's Sons, 1908, p. 37-42.

13. A. B. Walkley, Frames of Mind, Londres, Grant Richards, 1899, p. 119.

14. «Baedeker - Guidebooks as Books », The Times, 22 avril 1925.

15. F. Miltoun, Castles and Chateaux of Old Touraine and the Loire Country, Boston, L.C. Page, 1906, p. 3.

16. Ibid., p. 209 et 277.

17. E. Peixotto, Through the French Provinces, New York, C. Scribner's Sons, 1909, p. 107.

18. Ibid., p. 155.

19. T. A. Cook, Old Touraine: The Life and History of the Famous Chateaux of France, Londres, Percival and Co., 1892, vol. I, p. 30.

20. A. Mosher, The Spell of Brittany, New York, Duffield, 1920, p. 183-186.

21. F. Lees, A Summer in Touraine, Londres, Methuen, 1909, p. 96-97.

22. A. J. C. Hare, South-Western France, Londres, George Allen, 1890, p. 113-115 et p. 164-166.

23. Ibid., p. 166.

24. A. J. C. Hare, North-Western France, Londres, George Allen,1895, p. 212.

25. J. Tozier, A Spring Fortnight in France, Londres, T. Werner Laurie, 1907, p. 98.

26. C.N. Williamson et A.M. Williamson, The Lightning Conductor: The Strange Adventures of a MotorCar, New York, Grosset and Dunlap, 1903, p. 111-112.

27. A. B. Maurice, "The Literary Baedeker: Part II, Paris and Rural France ", The Bookman, juillet 1913, p. 546-563, ici p. 562.

28. E. G. Fay, « Balzac and Henry James », The French Review, vol. 24, nº 4, février 1951, p. 325-330.

29. H. James, "Honoré de Balzac", initialement publié dans The Galaxy en décembre 1875. Republié dans la collection French Poets and Novelists, Londres, Macmillan, 1878, p. 84-150.

30. W. W. Stowe, Balzac, James and the Realistic Novel, Princeton, Princeton University Press, 1983 ;

D. Gervais, "The Master's Lesson: Balzac and Henry James », The Cambridge Quarterly, 33(4), 2004, p. 315-330 ; S. Pietri, «Figures de l'œuvre-monde: "La Comédie humaine" de Henry James », L'Année balzacienne, 11(1), 2010, p. 365-440. 
31. H. James, art. cité, p. 115.

32. H. James, « Preface ", dans Roderick Hudson, New York, Charles Scribner's Sons, p. IX.

33. M.-R. Garnier, Henry James et la France, Paris, H. Champion, 1927, p. 106-123 ; J. DelbaereGarant, Henry James : The Vision of France, Liège, Presses universitaires de Liège, 1970.

34. P. Brooks, Henry James Goes to Paris, Princeton, Princeton University Press, 2018, p. 8.

35. H. James, Voyage en France, ouvr. cité, p. 21.

36. Ibid., p. 22.

37. Ibid., p. 24.

38. R. Burden, Travel, Modernism and Modernity, Farnham, Ashgate, 2015, p. 160.

39. H. James, English Hours, Londres, Heinemann, 1905, p. 44-49, p. 69, p. 199-201.

40. Ibid., p. 263.

41. H. James, Italian Hours, Boston, Houghton Mifflin, 1909, p. 466-67 et p. 329.

42. H. James, Voyage en France, ouvr. cité, p. 231.

43. Ibid., p. 344.

44. Ibid., p. 345.

45. Ibid., p. 29.

46. Ibid., p. 35.

47. D. Spurr, Architecture and Modern Literature, Ann Arbor, University of Michigan Press, 2012, p. 125.

48. H. James, Voyage en France, ouvr. cité, p. 103.

49. Ibid., p. 31.

50. Ibid., p. 181.

51. Ibid., p. 182.

52. H. James, Portraits of Places, Londres, Macmillan, 1883, p. 358.

53. H. James, Voyage en France, ouvr. cité, p. 116.

54. H. James, The Complete Letters of Henry James, 1880-1883, éd. Michael Anesko et Greg W. Zacharias, Lincoln, University of Nebraska Press, 2017, p. 220-221.

55. Ibid., p. 210-211.

56. Sur la carrière de Betham Edwards voir J. Rees, Matilda Betham Edwards: Novelist, Travel Writer and Francophile, Hastings, The Hastings Press, 2006 ; M. Cornick, «Matilda Betham-Edwards, Franco-British Go-between ", Synergies Royaume-Uni et Irlande, $\mathrm{n}^{\circ} 2$ 2, 2009, p. 55-68.

57. M. Betham Edwards, Holidays in Eastern France, Londres, Hurst and Blackett, 1879, p. vI.

58. M. Betham Edwards, Holiday Letters from Athens, Cairo and Weimar, Londres, Strahan and Co., 1873, p. 199.

59. M. Betham Edwards, A Year in Western France, Londres, Longmans, Green and Co., 1877.

60. « Western France », The Examiner, 24 février 1877, p. 241.

61. M. Betham Edwards, Reminiscences, Londres, George Redway, 1898, p. 192.

62. Ibid., p. 91 ; M. Betham Edwards, Anglo-French Reminiscences, Leipzig, Tauchnitz, 1900, p. 110 et p. 112.

63. M. Betham Edwards, Home Life in France, Londres, Methuen, 1905, p. 79 ; M. Betham Edwards, French, Men, Women and Books, Londres, Chapman and Hall, 1911, p. 43-92.

64. M. Betham Edwards, « On the Track of Balzac », The Sphere, 24 décembre 1904, p. 276.

65. M. Betham Edwards, Literary Rambles, ouvr. cité, p. 54-55.

66. M. Betham Edwards, In the Heart of the Vosges, Londres, Chapman and Hall, 1912, p. 141.

67. M. Betham Edwards, Literary Rambles, ouvr. cité, p. 50.

68. Ibid., p. 54-55.

69. Ibid., p. 55.

70. M. Betham Edwards, East of Paris, Londres, Hurst and Blackett, 1902, p. 79.

71. M. Betham Edwards, Literary Rambles, ouvr. cité, p. 49.

72. Ibid., p. 51. 
73. Ibid., p. 55.

74. Ibid., p. 50.

75. M. Betham Edwards, East of Paris, ouvr. cité, p. 79.

76. M. Betham Edwards, Literary Rambles, ouvr. cité, p. 106.

77. E. L. Robson, A Wayfarer on the Loire, Londres, Methuen, 1926.

\section{RÉSUMÉS}

Cet article analyse l'intérêt porté par les voyageurs anglophones aux œuvres de Balzac et aux sites qui leur sont associés. Se concentrant sur la période de 1880 à 1920, qui est marquée par l'essor du tourisme littéraire ainsi que par l'apogée de la réputation de Balzac dans le monde anglophone, il interroge la relation tissée, dans des récits de voyage sur la France provinciale, entre les textes littéraires, les lieux visités et l'expérience du voyageur. Il démontre que les attentes et les impressions de voyageurs tels que Henry James et Matilda Betham Edwards sont façonnées par leurs lectures, et ce à un tel point que les imaginaires littéraires associés au lieu suscitent parfois plus d'intérêt encore que la réalité.

This article examines the interest, among English-speaking travellers, in the works of Balzac and the places associated with them. Focusing on the period 1880-1920, which saw both the rise of the literary tourism and the peak of Balzac's reputation in the English-speaking world, it explores the relationship, in their travel writings on provincial France, between literary texts, places visited and the traveller's experience. It demonstrates that both the expectations and the experiences of travellers such as Henry James and Matilda Betham Edwards were shaped by their reading of literature, to the point that the literary associations of a place could become an even greater source of interest to them than the place itself.

\section{INDEX}

Mots-clés : tourisme littéraire, récit de voyage, lieux, lecteur, authenticité

Keywords : literary tourism, travel writing, places, reader, authenticity

\section{AUTEUR}

\section{TOM WILLIAMS}

Tom Williams est maître de conférences en civilisation britannique à l'université d'Angers. Docteur en histoire contemporaine de l'université d'Oxford et membre du Centre Interdisciplinaire de Recherche sur les Patrimoines en Lettres et Langues (CIRPALL, EA 7457), ses recherches portent sur l'histoire du tourisme, les usages politiques du passé, et les relations entre la Grande-Bretagne et l'Europe continentale. 